\title{
Precision Microstate Counting of Small Black Rings
}

\author{
Atish Dabholkar, ${ }^{1}$ Norihiro Iizuka, ${ }^{1}$ Ashik Iqubal, ${ }^{1}$ and Masaki Shigemori ${ }^{2}$ \\ ${ }^{1}$ Department of Theoretical Physics, Tata Institute of Fundamental Research, Mumbai 400 005, India \\ ${ }^{2}$ California Institute of Technology 452-48, Pasadena, California 91125, USA
}

(Received 4 December 2005; published 23 February 2006)

\begin{abstract}
We examine certain two-charge supersymmetric states with spin in five-dimensional string theories which can be viewed as small black rings when the gravitational coupling is large. Using the 4D-5D connection, these small black rings correspond to four-dimensional nonspinning small black holes. Using this correspondence, we compute the degeneracy of the microstates of the small black rings exactly and show that it is in precise agreement with the macroscopic degeneracy to all orders in an asymptotic expansion. Furthermore, we analyze the five-dimensional small black ring geometry and show qualitatively that the Regge bound arises from the requirement that closed timelike curves be absent.
\end{abstract}

PACS numbers: 11.25.Mj, 04.70.Dy, 11.25.Wx

Introduction. - One of the important successes of string theory has been that for a special class of supersymmetric black holes with a large classical area in the supergravity approximation, one can explain the Bekenstein-Hawking entropy in terms of statistical counting of their microstates [1]. Recently, it has become possible to extend these results to certain "small" black holes that have vanishing classical entropy but for which the quantum corrected entropy is nonzero and in precise agreement with the microscopic counting [2-8]. In fact, for these small black holes, a far more detailed comparison is possible and even the subleading corrections to the entropy are found to be in agreement with the state counting to all orders in a perturbative expansion in large charges. For this comparison to work, it is essential to include the quantum corrections to the Bekenstein-Hawking area formula itself in a systematic way $[9,10]$ using the attractor mechanism [11-16] and a specific statistical ensemble [17].

The black hole entropy thus provides a valuable clue about the microscopic structure of the theory. It is remarkable how tightly constrained the structure of string theory is. Various terms in the string effective action have to be of a very definite form with the right coefficients in order that the resulting macroscopic entropy matches with the counting of the microscopic quantum states of the theory to all orders.

In this Letter we generalize these results to states that carry spin. Spin introduces a number of qualitatively new features in the analysis of spacetime geometry especially in conjunction with supersymmetry. Supersymmetry requires that the angular velocity at the horizon be zero because otherwise there would be an ergoregion and energy can be extracted from the system for fixed charge in conflict with Bogomol'nyi-Prasad-Sommerfeld (BPS) stability. Hence for supersymmetric states, the angular momentum is typically swirling around outside the horizon. The nonspinning horizons, however, can have nontrivial geometry or topology. For example, adding spin to the D1-D5-P large black hole in five dimensions [1] deforms the round $\mathbf{S}^{3}$ horizon into an ellipsoid $[18,19]$. The area of the deformed horizon then correctly accounts for the modification of the entropy due to spin. For the same system, in a different regime of charge assignment, more exotic ringlike horizons with $\mathbf{S}^{2} \times \mathbf{S}^{1}$ topology are possible [20-24]. It is of interest therefore to know which of these possibilities would be realized for states that correspond to small black holes once spin is introduced and to ask if the counting still continues to be in agreement with the macroscopic entropy.

We consider spinning Dabholkar-Harvey (DH) states $[25,26]$ with two charges in toroidally compactified heterotic string theory in five dimensions. Using the chain of dualities and 4D-5D connection [27-30], it was shown in Ref. [31] that we can relate them to BPS states in four dimensions with four charges but no spin. This relation enables us to use the well-developed technology of the attractor equations including certain quantum corrections in four dimensions. One then finds that the resulting configuration has finite entropy after including the quantum corrections [31,32]. Moreover, as we will show, the microscopic and macroscopic entropy, in fact, agree to all orders in an asymptotic expansion.

Small black rings and 4D-5D connection. - Consider heterotic string compactified on $\mathbf{T}^{4} \times \mathbf{S}^{1}$ where $\mathbf{T}^{4}$ is a 4-torus in $\{6789\}$ directions and $\mathbf{S}^{1}$ is a circle along the $\{5\}$ direction. Consider now a string state with winding number $w$ along the $X^{5}$ direction. In a given winding sector, there is a tower of BPS states each in the right-moving ground state but carrying arbitrary left-moving oscillations subject to the Virasoro constraint $N_{L}=1+n w$, where $N_{L}$ is the leftmoving oscillation number and $n$ is the quantized momentum along $X^{5}[25,26]$. Note that $N_{L}$ is positive and hence a BPS state that satisfies this constraint has positive $n$ for positive $w$ for large $N_{L}$. This state can carry angular momentum $J$, say in the $\{34\}$ plane. The angular momentum operator $J$ is given by $J=\sum_{n=1}^{\infty}\left[a_{n}^{\dagger} a_{n}-\bar{a}_{n}^{\dagger} \bar{a}_{n}\right]$ where $a_{n}$ and $\bar{a}_{n}$ are the oscillator modes with frequency $n$ of the coordinates $\left(X^{3}+i X^{4}\right)$ and $\left(X^{3}-i X^{4}\right)$ respectively, normalized as $\left[a_{m}, a_{n}^{\dagger}\right]=\left[\bar{a}_{m}, \bar{a}_{n}^{\dagger}\right]=\delta_{m n}$.

Following the 4D-5D connection explained in Ref. [31] we can map this state to a configuration in Type IIA 
compactified on $\mathbf{K 3} \times \mathbf{T}^{2}$ with charges D2-D2-D0-D4 which in turn is dual to the DH states in heterotic string on $\mathbf{T}^{4} \times \mathbf{S}^{1} \times \tilde{\mathbf{S}}^{1}$ with momentum and winding $(n, w)$ and $(-\tilde{n}, \tilde{w})$ along the circles $\mathbf{S}^{1}$ and $\tilde{\mathbf{S}}^{1}$, respectively, with all integers $n, w, \tilde{n}, \tilde{w}$ positive. It is useful to state the 4D-5D connection entirely in the heterotic language. The basic idea following Refs. [27-30] is to make use of the Taub-Newman-Unti-Tamburino (Taub-NUT) geometry. For a Taub-NUT space with unit charge, the geometry near the origin is $\mathbf{R}^{4}$ whereas at asymptotic infinity it is $\mathbf{R}^{3} \times \tilde{\mathbf{S}}^{1}$. Thus a contractible circle at the origin of TaubNUT turns into a noncontractible circle $\tilde{\mathbf{S}}^{1}$ at asymptotic infinity. The angular momentum $J$ at the origin then turns into momentum $\tilde{n}$ along the circle at infinity [28].

Consider now DH states with spin in heterotic string theory on $\mathbf{T}^{4} \times \mathbf{S}^{1}$. Spinning strings that are wrapping along the circle $\mathbf{S}^{1}$ in $\{5\}$ direction and rotating in the $\{34\}$ plane have a helical profile [33-36]. The helix goes around a contractible circle $\mathbf{S}_{\psi}^{1}$ of radius $R_{\psi}$ along an angular coordinate $\psi$ in the $\{34\}$ plane as the string wraps around the noncontractible circle $\mathbf{S}^{1}$. Let us denote the pitch of the helix by $p$, which is the winding number of the projection of the helix onto the contractible circle. Macroscopically it corresponds to a dipole charge. We can now embed this system in Taub-NUT space with very large Taub-NUT radius $R_{\mathrm{TN}} \gg R_{\psi}$ and regard $\mathbf{S}_{\psi}^{1}$ in $\mathbf{R}^{4}$ as being situated at the origin of a Taub-NUT geometry. Varying the radius of Taub-NUT, which is a modulus, we can smoothly go to the regime $R_{\mathrm{TN}} \ll R_{\psi}$. Then the contractible $\mathbf{S}_{\psi}^{1}$ effectively turns into the noncontractible circle $\tilde{\mathbf{S}}^{1}$ at asymptotic infinity. The entropy of BPS states is not expected to change under such an adiabatic change of moduli. We can dimensionally reduce the system to 4D along $\tilde{\mathbf{S}}^{1}$ and obtain a 4D DH state with four charges $(n, w,-\tilde{n}, \tilde{w})$ with the identification that $\tilde{n}=J$ and $\tilde{w}=$ $p$. This system has a string scale horizon in 4D [2-8] which suggests that the original spinning DH system in $5 \mathrm{D}$ is a small black ring [31].

Since we have unit Taub-NUT charge to begin with, we do not have a purely electric configuration in $4 \mathrm{D}$ but instead have a Kaluza-Klein (KK) monopole of unit charge in addition to the 4-charge purely electric small black hole. However, since the helix is far away from the origin of Taub-NUT space in 5D before dimensional reduction, the KK monopole is sitting far away from the 4-charge black hole in 4D. The separation is determined by Denef's constraint [37] and is determined by $J$ and the asymptotic values of the moduli and can thus be made arbitrarily large. The local microscopic counting therefore does not depend on the addition of the KK monopole and is given by the counting of DH states.

Four-dimensional counting. - In the 4D description, the state is specified by the charge vector $Q$ in the Narain charge lattice $\Gamma^{2,2}$ of the $\mathbf{S}^{1} \times \tilde{\mathbf{S}}^{1}$ factor with four integer entries. The norm of this vector is

$$
\frac{Q^{2}}{2}=\frac{1}{2}\left(\begin{array}{llll}
n & w & -\tilde{n} & \tilde{w}
\end{array}\right)\left(\begin{array}{cccc}
0 & 1 & 0 & 0 \\
1 & 0 & 0 & 0 \\
0 & 0 & 0 & 1 \\
0 & 0 & 1 & 0
\end{array}\right)\left(\begin{array}{c}
n \\
w \\
-\tilde{n} \\
\tilde{w}
\end{array}\right) .
$$

The degeneracy of these perturbative DH states can be computed exactly and the asymptotic degeneracy for large $Q^{2}$ is given as in Refs. [4,5] by

$$
\Omega_{\text {micro }}(n, w, \tilde{n}, \tilde{w}) \sim \hat{I}_{13}\left(4 \pi \sqrt{\frac{Q^{2}}{2}}\right)=\hat{I}_{13}(4 \pi \sqrt{n w-\tilde{n} \tilde{w}}),
$$

where $\hat{I}_{13}(z)$ is the modified Bessel function defined in Ref. [5].

Turning to the macroscopic degeneracy, we compute it using the Ooguri-Strominger-Vafa (OSV) relation [17] between topological string partition function and the macroscopic degeneracy, for the nonspinning four-dimensional configuration. For this purpose we use the Type IIA description, viewing this state as a collection of D2-D2-D0$\mathrm{D} 4$ branes. There are $n \mathrm{D} 2$-branes wrapping a 2-cycle $\alpha_{1}$ in $\mathbf{K 3}$ and $w$ D2-branes wrapping a 2-cycle $\alpha_{2}$ such that the intersection matrix of $\alpha_{1}$ and $\alpha_{2}$ is as in the upper-left $2 \times$ 2 block of the matrix in (1). We therefore identify the charges as $\left(q_{2}, q_{3}\right)=(n, w)$. Similarly, we identify the D0-D4 charges as $\left(q_{0}, p^{1}\right)=(\tilde{n}, \tilde{w})$ so in the labeling of charges used in Ref. [5], we have $Q=\left(q_{2}, q_{3}, q_{0}, p^{1}\right)$ and all other charges zero. Using the formula (2.26) in [5] we then see that the macroscopic degeneracy is given by

$$
\begin{aligned}
\Omega_{\text {macro }}(n, w, J) & \sim\left(p^{1}\right)^{2} \hat{I}_{13}\left(4 \pi \sqrt{q_{2} q_{3}-p^{1} q_{0}}\right) \\
& =\left(p^{1}\right)^{2} \hat{I}_{13}(4 \pi \sqrt{n w-\tilde{n} \tilde{w}}) .
\end{aligned}
$$

Therefore, up to the overall $\left(p^{1}\right)^{2}$ factor, the microscopic (2) and macroscopic (3) degeneracies match precisely to all orders in an asymptotic expansion for large $Q^{2}$.

Five-dimensional microscopic counting.-We now would like to count the degeneracy of the spinning DH system from the $5 \mathrm{D}$ side. The nontrivial issue is to determine the correct ensemble. The relevant states correspond to quantum fluctuations around a specific coherent oscillating state which is essentially Bose-Einstein condensate on the world sheet and describes the helical geometry with pitch $p$ [31]. As we will argue below, the precise microstates turn out to be of the form

$$
\underbrace{\left(a_{p}^{\dagger}\right)^{J}}_{\begin{array}{c}
\text { microscopic origin of } \\
\tilde{n}=J \text { and } \tilde{w}=p \text { of } \\
\text { thering }
\end{array}} \times \underbrace{\prod_{n=1}^{\infty}\left[\prod_{i=1,2, \pm, 5 \ldots 24}\left(\alpha_{-n}^{i}\right)^{N_{n i}}\right]|0\rangle}_{\begin{array}{c}
\text { fluctuation: all possiblestates } \\
\text { withlevel } N_{\text {eff }} \equiv N-p J \text {. Angular } \\
\text { momentum } J \text { is notfixed. }
\end{array}}
$$

for $J, p>0$. Namely, we consider the states with the world sheet energy $N_{\text {eff }} \equiv N-p J$ and chemical potential $\mu$ 
conjugate to $J$ set to zero, and multiply all those states by $\left(a_{p}^{\dagger}\right)^{J}$. Note, in particular, that the $a_{p}^{\dagger}$ and $\bar{a}_{p}^{\dagger}$ oscillators are included in the fluctuation part. The degeneracy of the states (4) is the same as that of the DH system with $Q^{2} / 2=$ $N_{\text {eff }}$ and proportional to $\hat{I}_{13}(4 \pi \sqrt{N-p J})$, in precise agreement with (2) and (3). If $J, p<0,\left(a_{p}^{\dagger}\right)^{J}$ in (4) must be replaced by $\left(\bar{a}_{|p|}^{\dagger}\right)^{|J|}$.

This separation between the classical coherent condensate that describes the large helix and the small quantum fluctuations around it that account for the entropy is similar to the one used in Refs. [38-42]. It is valid in the regime when $R_{\psi}$ is much larger than the amplitude of fluctuation. Therefore, we conclude that in this regime, the states of the form (4) are the states that account for the microstates of the ring. This in turn agrees with (2) and (3) in 4D through the 4D-5D connection.

Note that (4) means that the microscopic counting in 5D must not be done for fixed angular momentum $J$. Fixing $J$ would impose an additional constraint on the fluctuation part in (4). From the 4D point of view, it would correspond to imposing a constraint on the world sheet oscillators of the DH system, which would lead to a result contradictory to the 4D degeneracy (2) and (3). To demonstrate this, let us count the degeneracy of spinning DH states with fixed $J$. The degeneracy $\Omega(N, J)$ is summarized in the partition function

$$
Z(\beta, \mu)=\sum_{N, J} \Omega(N, J) q^{N} c^{J}, \quad q=e^{-\beta}, \quad c=e^{\beta \mu},
$$

where $N \equiv n w=N_{L}-1$. $\beta$ can be thought of as the inverse temperature on the world sheet for a $1+1$ gas of left-moving 24 bosons conjugate to the total energy $N$ and $\mu$ can be thought of as the chemical potential conjugate to the quantum number $J$ of this gas. Since $N_{L}$ is the oscillation number for the 24 left-moving transverse bosons, using the expression $J=\sum_{n=1}^{\infty}\left[a_{n}^{\dagger} a_{n}-\bar{a}_{n}^{\dagger} \bar{a}_{n}\right]$, the partition function can be readily evaluated [43] and is given by

$$
\begin{aligned}
Z(\beta, \mu) & =\left[q \prod_{n=1}^{\infty}\left(1-q^{n}\right)^{22}\left(1-c q^{n}\right)\left(1-c^{-1} q^{n}\right)\right]^{-1} \\
& =\frac{1}{\eta^{21}\left(e^{-\beta}\right)} \frac{2 i \sinh (\beta \mu / 2)}{\theta_{11}(\beta \mu / 2 \pi i, i \beta / 2 \pi)},
\end{aligned}
$$

in terms of the standard Dedekind eta function and theta function with characteristics.

The number of states with given $N$ and $J$ is then given by the inverse Laplace transform: $\Omega(N, J)=\frac{1}{(2 \pi i)^{2}} \times$ $\int_{C_{\beta}} d \beta e^{\beta N} \int_{C_{\mu}} \beta d \mu e^{-\mu \beta J} Z(\beta, \mu)$, where the contour $C_{\beta}$ runs from $-i \pi+\gamma$ to $+i \pi+\gamma$ with $\gamma>0$ to avoid singularities on the imaginary axis. Similarly, $C_{\mu}$ goes from $-\pi i / \beta+\epsilon$ to $+\pi i / \beta+\epsilon$ with $-1<\epsilon<1$ to avoid poles. To find the asymptotic degeneracy at large $N$, we want to take the high temperature limit, or $\beta \rightarrow 0$. Using the modular properties of the Dedekind eta and the theta functions we can write the degeneracy at high temperature as in [43] as

$$
\Omega(N, J) \sim \frac{1}{2 \pi i} \int_{C_{\beta}} d \beta e^{\beta N+(2 \pi)^{2} / \beta}\left(\frac{\beta}{2 \pi}\right)^{12} I(\beta, J),
$$

where $I(\beta, J)$ is defined by

$$
I(\beta, J)=\frac{1}{2 \pi i} \int_{C_{\mu}} d \mu e^{-\beta \mu^{2} / 2-\beta \mu J} \frac{\sinh (\beta \mu / 2)}{\sin (\pi \mu)} .
$$

To arrive at (7), we dropped terms that are exponentially suppressed for small $\beta$ as $e^{-(2 \pi)^{2} / \beta}$. This is justified although $\beta$ is still to be integrated over, because the saddle point around $\beta \sim 1 / \sqrt{N-J} \ll 1$ will make the leading contribution, as we will see below.

Now we evaluate (8) using the method of residues. Deform the contour $C_{\mu}$ into sum of three intervals $C_{1}=$ $[-\pi i / \beta+\epsilon,-\pi i / \beta+K], C_{2}=[-\pi i / \beta+K, \pi i / \beta+$ $K]$, and $C_{3}=[\pi i / \beta+K, \pi i / \beta+\epsilon]$, with $K \gg 1$. One can readily show that the contour integral along $C_{1,2,3}$ vanishes due to the periodicity of the original integrand (6) in the small $\beta$ and large $K$ limit. In the process of deforming the contour, we pick up poles at $\mu=m, m=$ $1,2, \ldots$ In the end, we obtain

$$
\begin{aligned}
I(\beta, J) & \sim-\operatorname{Res}_{\mu=1}\left[e^{-\beta \mu^{2} / 2-\beta \mu J} \frac{\sinh (\beta \mu / 2)}{\sin (\pi \mu)}\right]+\mathcal{O}\left(e^{-2 \beta J}\right) \\
& \sim\left(1-e^{-\beta}\right) e^{-\beta J}+\mathcal{O}\left(e^{-2 \beta J}\right) .
\end{aligned}
$$

Here $\mathcal{O}\left(e^{-2 \beta J}\right)$ comes from the poles at $\mu=2,3, \ldots$ and is negligible when $J=\mathcal{O}(N)$ since $\beta J=\mathcal{O}\left(N^{1 / 2}\right)$. We interpret the term $\propto e^{-\beta J}$ as the contribution from the $p=$ 1 sector. Substituting this back into (7), we conclude that the degeneracy $\Omega(N, J)$ is $\sim \frac{1}{2 \pi i} \int d \beta(\beta / 2 \pi)^{12}(\beta-$ $\left.\frac{\beta^{2}}{2}+\cdots\right) e^{(2 \pi)^{2} / \beta+\beta(N-J)}$. Each term in the integral is of the Bessel type as discussed in Ref. [5], and thus the final result is $\Omega(N, J) \sim \hat{I}_{14}(4 \pi \sqrt{N-J})-(2 \pi / 2) \times$ $\hat{I}_{15}(4 \pi \sqrt{N-J})+\cdots$, which agrees with (2) and (3) with $p=1$ only in the leading exponential but disagrees in the subleading corrections. This demonstrates that microscopic counting in 5D must be done not for fixed $J$ but for the states (4). One can show that states with fixed $\mu \neq$ 0 also lead to degeneracy in disagreement with (2) and (3).

In general, subleading corrections to thermodynamic quantities depend on the choice of the statistical ensembles and are different for different ensembles. For example, even for nonspinning black holes the ensemble with fixed angular momentum $J=0$ differs from the ensemble with fixed chemical potential $\mu=0$ in subleading corrections. It was noted in [5] that the correct microscopic ensemble that is consistent with the OSV conjecture is the one with $\mu=0$. In our case, the description of small black rings requires that we also fix the pitch of the helix as an additional requirement and that we are counting states around this classical coherent condensate on the world sheet. 
Five-dimensional macroscopic geometry. -Finally, we comment on the geometry of this 5D small black ring, which can be determined in the near ring limit by using the 4D-5D uplift. Exact uplift is possible near the horizon, since the near-horizon geometry of 4D black hole can be determined precisely by using string-corrected attractor equations. Let us consider the case where $p J=\mathcal{O}(N)$. We consider a helix with general pitch $p$ even though the contribution from the $p=1$ subsector dominates entropy. In $4 \mathrm{D}$ heterotic string theory, the near-horizon black hole geometry is determined by the attractor equations to be $e^{2 \phi_{4}} \sim 1 / \sqrt{N-\bar{J}}, \sqrt{g_{\psi \psi}} \sim \sqrt{J / p}$, and the horizon radius is $r_{\mathbf{S}^{2}} \sim l_{s}$. Using $e^{2 \phi_{5}} \sim e^{2 \phi_{4}} \sqrt{g_{\psi \psi}}$ and $l_{s} \sim e^{-2 \phi_{5} / 3} l_{p l}^{(5)}$, the scale of $\mathbf{S}_{\psi}^{1}$ along ring is given by

$$
\begin{aligned}
& r_{\mathbf{S}^{1}} \sim \sqrt{g_{\psi \psi}} l_{s} \sim p^{-1 / 3} J^{1 / 3}(N-p J)^{1 / 6} l_{p l}^{(5)}, \\
& r_{\mathbf{S}^{2}} \sim l_{s} \sim p^{1 / 6} J^{-1 / 6}(N-p J)^{1 / 6} l_{p l}^{(5)},
\end{aligned}
$$

such that $S \sim A / 4 G_{5} \sim r_{\mathbf{S}^{1}}\left(r_{\mathbf{S}^{2}}\right)^{2} /\left(l_{p l}^{(5)}\right)^{3} \sim \sqrt{N-p J}$. One important qualitative feature of the solution is that when $p J$ exceeds $N$, the solution develops closed timelike curves. When $N \geq p J$ is saturated, $g_{\psi \psi} \geq 0$ is saturated at the ring horizon. Hence, the Regge bound $N \geq p J$ on the angular momentum of the underlying microstates can be understood from the macroscopic solution as a consequence of the physical requirement that closed timelike curves be absent. The details will be presented elsewhere [44].

We are very grateful to Ashoke Sen for numerous illuminating discussions and collaboration on Ref. [44]. A. D. would like to thank the high energy group at ASICTP, Trieste for their hospitality where part of this work was completed. The work of M.S. was supported in part by Department of Energy Grant No. DE-FG03-92ER40701 and the Sherman Fairchild Foundation.

[1] A. Strominger and C. Vafa, Phys. Lett. B 379, 99 (1996).

[2] A. Dabholkar, Phys. Rev. Lett. 94, 241301 (2005).

[3] A. Dabholkar, R. Kallosh, and A. Maloney, J. High Energy Phys. 12 (2004) 059.

[4] A. Dabholkar, F. Denef, G. W. Moore, and B. Pioline, J. High Energy Phys. 08 (2005) 021.

[5] A. Dabholkar, F. Denef, G. W. Moore, and B. Pioline, hepth/0507014

[6] A. Sen, Mod. Phys. Lett. A 10, 2081 (1995).

[7] A. Sen, J. High Energy Phys. 05 (2005) 059.

[8] A. Sen, hep-th/0504005.

[9] R. M. Wald, Phys. Rev. D 48, R3427 (1993).

[10] V. Iyer and R. M. Wald, Phys. Rev. D 50, 846 (1994).

[11] S. Ferrara, R. Kallosh, and A. Strominger, Phys. Rev. D 52, R5412 (1995).
[12] G. Lopes Cardoso, B. de Wit, and T. Mohaupt, Phys. Lett. B 451, 309 (1999).

[13] G. Lopes Cardoso, B. de Wit, and T. Mohaupt, Fortschr. Phys. 48, 49 (2000).

[14] G. Lopes Cardoso, B. de Wit, and T. Mohaupt, Classical Quantum Gravity 17, 1007 (2000).

[15] G. Lopes Cardoso, B. de Wit, J. Kappeli, and T. Mohaupt, J. High Energy Phys. 12 (2000) 019.

[16] J. M. Maldacena, A. Strominger, and E. Witten, J. High Energy Phys. 12 (1997) 002.

[17] H. Ooguri, A. Strominger, and C. Vafa, Phys. Rev. D 70, 106007 (2004).

[18] J. C. Breckenridge, R. C. Myers, A. W. Peet, and C. Vafa, Phys. Lett. B 391, 93 (1997).

[19] J. P. Gauntlett, R. C. Myers, and P. K. Townsend, Classical Quantum Gravity 16, 1 (1999).

[20] H. Elvang, R. Emparan, D. Mateos, and H. S. Reall, Phys. Rev. Lett. 93, 211302 (2004).

[21] H. Elvang, R. Emparan, D. Mateos, and H. S. Reall, Phys. Rev. D 71, 024033 (2005).

[22] J.P. Gauntlett and J.B. Gutowski, Phys. Rev. D 71, 025013 (2005).

[23] J.P. Gauntlett and J.B. Gutowski, Phys. Rev. D 71, 045002 (2005).

[24] I. Bena and N. P. Warner, hep-th/0408106.

[25] A. Dabholkar and J. A. Harvey, Phys. Rev. Lett. 63, 478 (1989).

[26] A. Dabholkar, G. W. Gibbons, J. A. Harvey, and F. Ruiz Ruiz, Nucl. Phys. B340, 33 (1990).

[27] D. Gaiotto, A. Strominger, and X. Yin, hep-th/0503217.

[28] H. Elvang, R. Emparan, D. Mateos, and H.S. Reall, J. High Energy Phys. 08 (2005) 042.

[29] D. Gaiotto, A. Strominger, and X. Yin, hep-th/0504126.

[30] I. Bena, P. Kraus, and N.P. Warner, Phys. Rev. D 72, 084019 (2005).

[31] N. Iizuka and M. Shigemori, J. High Energy Phys. 08 (2005) 100.

[32] K. Behrndt, G. L. Cardoso, and S. Mahapatra, Nucl. Phys. B732, 200 (2006).

[33] A. Dabholkar, J. P. Gauntlett, J. A. Harvey, and D. Waldram, Nucl. Phys. B474, 85 (1996).

[34] C. G. Callan, J. M. Maldacena, and A. W. Peet, Nucl. Phys. B475, 645 (1996).

[35] O. Lunin and S. D. Mathur, Nucl. Phys. B610, 49 (2001).

[36] O. Lunin and S. D. Mathur, Phys. Rev. Lett. 88, 211303 (2002).

[37] F. Denef, J. High Energy Phys. 08 (2000) 050.

[38] B. C. Palmer and D. Marolf, J. High Energy Phys. 06 (2004) 028.

[39] D. Marolf and B.C. Palmer, Phys. Rev. D 70, 084045 (2004).

[40] I. Bena and P. Kraus, J. High Energy Phys. 12 (2004) 070.

[41] P. Kraus and F. Larsen, J. High Energy Phys. 09 (2005) 034.

[42] D. Bak, Y. Hyakutake, S. Kim, and N. Ohta, Nucl. Phys. B712, 115 (2005).

[43] J. G. Russo and L. Susskind, Nucl. Phys. B437, 611 (1995).

[44] A. Dabholkar, N. Iizuka, A. Iqubal, A. Sen, and M. Shigemori (to be published). 